Ce qu'un instrument fait à la gouvernance locale. Les contrats de concession et la régulation de la distribution publique d'électricité en France (1989-2015)

How an instrument shapes local governance. Concession contracts and the regulation of electricy supply in France (1989-2015)

\title{
François-Mathieu Poupeau
}

\section{OpenEdition}

Journals

Édition électronique

URL : http://journals.openedition.org/pmp/9350

ISSN : 2119-4831

Éditeur

Institut de Management Public (IDPM)

Édition imprimée

Date de publication : 1 juillet 2015

Pagination : 211-240

ISBN : $978-2-7430-2138-2$

ISSN : 0758-1726

Référence électronique

François-Mathieu Poupeau, « Ce qu'un instrument fait à la gouvernance locale. Les contrats de concession et la régulation de la distribution publique d'électricité en France (1989-2015) », Politiques et management public [En ligne], Vol 32/3 | 2015, mis en ligne le 21 juin 2019, consulté le 24 janvier 2021. URL : http://journals.openedition.org/pmp/9350 


\author{
François-Mathieu Poupeau \\ Chercheur CNRS au LATTS (Université Paris-Est)
}

\title{
Résumé
}

Qu'est-ce qu'un instrument peut faire à l'action publique locale et à la manière dont elle est gouvernée et pilotée? C'est en substance la question qui est posée dans cet article consacré à la négociation, dans les années 1990, de nouveaux traités de concession de distribution publique d'électricité en France. Utilisant la notion de script développée par Madeleine Akrich, l'article montre comment des acteurs - en l'occurrence EDF et les élus ruraux - ont su utiliser l'outil contractuel au service d'un processus d'« ingénierie territoriale » visant à les protéger face aux menaces que leur faisait courir le processus de libéralisation. Dans cette perspective, le contrat n'apparaît plus comme un simple dispositif organisant des rapports de coopération entre partenaires. En contribuant à façonner les règles du jeu qui structurent la régulation d'un secteur, il se révèle être un puissant opérateur de réhiérarchisation institutionnelle, dont les effets se font fortement ressentir aujourd'hui, à l'heure où des réflexions émergent autour d'une nouvelle gouvernance territoriale de la transition énergétique, davantage ancrée dans les métropoles.

(c) 2015 IDMP/Lavoisier SAS. Tous droits réservés

Mots clés : contrat, instrument, électricité, libéralisation, gouvernance, script.

\section{Abstract}

How an instrument shapes local governance. Concession contracts and the regulation of electricy supply in France (1989-2015). How does an instrument, namely a contract, influence local public action and the way it is governed and steered? This is the core issue of this article, which deals with the effects of the negotiation, in the 1990s, of new concession contracts between EDF and local authorities in France, the latter owning public electricity networks. The author uses the notion of "script", developed by Madeleine Akrich, to show how EDF and rural politicians catched the contractual instrument as a means to

\footnotetext{
*Auteur correspondant : fm.poupeau@enpc.fr

doi :10.3166/pmp.32.211-240 @ 2015 IDMP/Lavoisier SAS. Tous droits réservés
} 
protect themselves against the liberalization process. In such a perspective, contract is not simply a tool which organizes cooperative relationships between partners. It appears also as an instrument which can shape new institutional hierarchies, by designing new rules of the game. Such an action has long-term effects on local governance, especially in the current context of energy transition and metropolisation. @ 2015 IDMP/Lavoisier SAS. Tous droits réservés

Keywords: contract, instrument, electricity, liberalization, governance, script.

\section{Introduction}

À la faveur du processus de libéralisation et de la mise sur l'agenda politique de la notion de transition énergétique, les élus locaux français ont redécouvert une compétence historique qui avait été largement occultée par un demi-siècle d'intervention de l'État : le statut d'autorité concédante des réseaux de distribution publique d'électricité dont jouissent les communes et leurs groupements (Poupeau, 2004). Institué par une loi déjà centenaire mais sujette à plusieurs adaptations, il confère aux pouvoirs locaux des prérogatives importantes en matière d'acheminement des flux d'énergie électrique, la partie fourniture faisant quant à elle l'objet d'une régulation de marché où intervient essentiellement l'État ${ }^{1}$. Les élus disposent en effet d'un droit de regard sur les investissements réalisés dans les réseaux desservant leur territoire, ainsi que sur une part des tarifs entrant dans la composition du prix de l'électricité payé par les usagers domestiques². À l'heure où les réseaux d'énergie occupent une place importante dans le processus de transition énergétique (smart grids, raccordement des énergies renouvelables...), la redécouverte de ce statut a suscité de nombreuses prises de position contradictoires voire des polémiques au sein du monde des collectivités locales. Elles ont notamment trouvé à s'exprimer lors du Débat national sur la transition énergétique (DNTE) lancé par le gouvernement français de 2012 à l'été 2013 pour préparer la loi sur la transition énergétique et la croissance verte ${ }^{3}$. Conçu comme un

\footnotetext{
${ }^{1}$ La compétence communale date de la loi du 15 juin 1906, qui assimile alors la distribution-fourniture d'énergie électrique à un service public local. Dans les décennies qui suivent, notamment après la nationalisation et la création d'EDF, la loi de 1906 perd de fait de son contenu, les investissements et surtout les tarifs étant désormais gérés essentiellement à l'échelle nationale. Mais la concession porte toujours, en théorie, sur les deux dimensions de distribution et de fourniture. Les évolutions qui ont cours dans les années 1990 et 2000 confortent quelque peu ce glissement progressif, réduisant le champ d'intervention des élus à la partie distribution (Poupeau, 2004). En effet, exception faite des tarifs réglementés, qui restent formellement dans le périmètre des contrats de concession, c'est l'État qui a en charge la régulation tarifaire, les communes étant clairement marginalisées sur ce plan. Dans la suite du texte, nous utiliserons deux termes pour bien marquer ce rétrécissement progressif du champ d'action de la concession : distribution-fourniture s'agissant de la période avant la libéralisation (avec une concession englobant l'acheminement et la fourniture) et distribution (acheminement) lorsqu'est mis en œuvre le principe de la séparation des deux activités (ou unbundling), avec mise en concurrence de la partie " fourniture ».

${ }^{2}$ Ce qu'on appelle le tarif d'utilisation des réseaux publics d'électricité (TURPE), qui correspond, pour résumer, aux frais de « péage » dont doivent s'acquitter les consommateurs qui empruntent les réseaux de transport et de distribution. À I'heure actuelle, le TURPE représente environ un tiers de la facture totale acquittée par un usager domestique (les deux tiers restants concernant la fourniture et les taxes et redevances diverses). 3 Sur ces prises de position, cf Comité national du débat sur la transition énergétique (CNDTE), «Les cahiers d'acteurs », Le dossier du débat national sur la transition énergétique, mai 2013.
} 
lieu d'échange élargi, le DNTE a notamment révélé les aspirations nouvelles des villes et métropoles à se saisir de cette compétence pour se positionner comme des acteurs à part entière de la transition énergétique. Il a surtout mis en exergue les nombreuses difficultés associées à une telle entreprise de réappropriation, difficultés qui sont à rechercher tant du côté d'EDF-ERDF ${ }^{4}$ que de la position hégémonique occupée sur la scène institutionnelle locale par les représentants du monde rural, lesquels, via des syndicats départementaux bien implantés, ont œuvré pour préserver un modèle de service public qu'ils souhaitent conserver unitaire et national.

Cette difficulté à réorienter la gouvernance locale des réseaux de distribution publique d'électricité tient en large part - et c'est là l'objet de cet article - aux effets d'un processus qui s'est déroulé tout au long de la décennie 1990, à l'ombre des grandes négociations menées entre l'État français et l'Union européenne autour du processus de libéralisation : la signature de nouveaux contrats de concession entre EDF et les communes françaises. $\mathrm{Au}$ moment même où la France s'engage non sans réticence dans la voie de l'ouverture à la concurrence de son marché domestique (Stoffaes, 1994), EDF entreprend alors de combler le vide juridique qui existait depuis le vote de la loi de nationalisation, la plupart des traités issus de la loi de 1906 n'ayant jamais été révisés. Ce processus ne constitue pas qu'une simple opération de « toilettage » juridique, neutre au plan de l'organisation institutionnelle (mettre à jour des traités de concession déjà anciens). Il contribue à transformer l'architecture des pouvoirs et des modes de régulation du secteur. L'usage du contrat permet en effet à l'entreprise publique de geler durablement toute tentative de remise en cause du monopole dont elle dispose alors en matière de distribution-fourniture d'électricité. Il est également mobilisé par les élus ruraux pour défendre, à travers le renforcement du système départemental, les intérêts de territoires qui se sentent menacés par une politique de libéralisation pouvant, à leurs yeux, profiter aux grandes villes.

C'est à ce travail de « formatage » de la régulation territoriale de la distribution publique d'électricité par un instrument - le contrat de concession - qu'est consacré cet article. Ici, le contrat n'est pas envisagé comme un document régissant des rapports de forces et de coopération entre des agents économiques dont l'existence est considérée comme une donnée de départ, intangible, à l'instar des économistes ou des sociologues qui s’intéressent aux questions d'expertise et d'asymétrie d'informations (Akerlof, 1970 ; Spence, 1973; Stiglitz, Weiss, 1981; Laffont, Tirole, 1993), d'incertitudes tenant au caractère incomplet du contrat (Hart, Moore, 1988; Barnett, 1992) ou de phénomènes de confiance (Neuville, 1996; Brousseau, 2000). Il est plutôt appréhendé comme un instrument d'action publique (Lascoumes, Le Galès, 2004 ; Lascoumes, Simard, 2011 ; Halpern, Lascoumes, Le Galès, 2014) mobilisé par des acteurs souhaitant structurer un secteur d'activité en ordonnançant le paysage institutionnel qui le régule. Le contrat apparaît alors comme faisant partie d'une panoplie d'outils plus larges dont les acteurs peuvent se saisir pour agencer le marché, tant du côté des entreprises que des pouvoirs publics : textes de lois, décrets, circulaires, systèmes de tarification, décisions régulatoires... Il véhicule des choix institutionnels

\footnotetext{
${ }^{4}$ À qui il est reproché une certaine opacité dans la gestion des réseaux de distribution publique d'électricité ainsi que l'opposition à toute forme d'évolution vers un système plus décentralisé, qui pourrait même permettre à certaines communes de municipaliser la gestion de ce service public, à l'instar du mouvement observé dans d'autres secteurs (l'eau) ou d'autres pays (Allemagne).
} 
implicites, en jouant sur le design de la régulation, en mettant en avant certains acteurs, en privilégiant des périmètres d'action, bref en organisant les conditions dans lesquelles les agents économiques vont être par la suite amenés à entrer dans l'arène de la négociation.

Afin de montrer ce rôle joué par l'instrument contractuel, nous procéderons en deux temps, en nous appuyant sur un corpus d'information nourri de recherches régulières sur le secteur de l'électricité . Nous nous intéresserons tout d'abord à un moment clé du processus de contractualisation, à savoir l'élaboration, au tournant des décennies 1980 et 1990, d'un modèle de cahier des charges destiné à servir de support aux relations entre le concessionnaire EDF et ses autorités concédantes, les communes (parties I à III). Pour ce faire, nous utiliserons la notion de «script » telle qu'elle a été théorisée par Madeleine Akrich. Madeleine Akrich appelle un «script» « un « scénario » qui se veut prédétermination des mises en scène que les utilisateurs sont appelés à imaginer à partir du dispositif technique et des pré-scriptions (notices, contrats, conseils...) qui l'accompagnent» (Akrich, 1987 : 52). Matérialisé par des clauses juridiques et des modes de calculs financiers (montant de certaines redevances) incorporés dans le modèle de cahier des charges proposé aux communes, ce «script» se caractérise par un objectif politique sous-jacent : préserver un modèle de service public national en incitant les communes à se regrouper au plan départemental. Dans un second temps (partie IV), l'article s'intéressera au moment où ce «script» initial est mis à l'épreuve, à travers les controverses et formes d'opposition qui se font jour quand le modèle de cahier des charges est diffusé sur l'ensemble du territoire national. Le contrat n'a en effet pas de force intrinsèque. La performativité du script dépend des conditions de son déploiement. S'engage alors ce que nous appellerons un processus d' « ingénierie institutionnelle » qui fait intervenir les agents d'EDF ainsi que des représentants des élus ruraux. Leur travail consiste à « enrôler » les acteurs locaux, à les inciter à signer le nouveau modèle de cahier des charges afin de faire advenir le scenario institutionnel que ce document sous-tend. Ce processus se déploie tout au long des années 1990 et 2000, non sans un certain succès car il aboutit à promouvoir une départementalisation de la gouvernance locale des réseaux d'électricité que remettent actuellement en cause certains élus, au sein notamment des métropoles (Poupeau, 2015b).

\section{La libéralisation, une menace pour le système de distribution-fourniture d'électricité français}

La négociation des contrats de concession entre EDF et les collectivités locales françaises s'inscrit dans un contexte particulier, le début des années 1990 et la perspective d'une mise en concurrence des activités de distribution-fourniture d'énergie, jusque-là assurées de manière intégrée par un grand monopole public (EDF). Initiée à la fin des années 1980 suite à la relance du processus d'intégration européenne (Stoffaes, 1994), la libéralisation met alors en lumière une dimension du modèle français de service public quelque peu oubliée - hormis de certains spécialistes avertis - depuis la loi de nationalisation de 1946 : le fait que la distribution-fourniture d'électricité relève en partie de la compétence communale. Conjuguée à la décentralisation, qui a revigoré les pouvoirs locaux, cette redécouverte menace de déstabiliser les acteurs du modèle français de service public, EDF en tête mais

\footnotetext{
${ }^{5}$ Le lecteur se reportera à l'annexe 1 pour plus de précisions sur la nature du matériau utilisé.
} 
aussi les élus ruraux, dont les intérêts et revendications avaient été largement pris en compte dans la période suivant la nationalisation.

\subsection{La mise sur l'agenda d'une politique de mise en concurrence}

Très importante en France jusqu' aux années 1960, date à laquelle elle tend quelque peu à s'effacer (Poupeau, 1999), la place des collectivités locales dans la gestion du service public de l'électricité resurgit à la toute fin des années 1980, lorsque se dessine la perspective de constitution d'un grand marché européen de l'énergie, sur fond de libéralisation. À vrai dire, l'intégration européenne ne saurait être considérée en elle-même comme le déclencheur d'une stratégie de mise en concurrence des monopoles publics. Elle en constitue plutôt un vecteur. En France, la remise en cause d'EDF se fait en effet sentir dès les années 1970, décennie de contestation de la toute-puissance de l'industrie nucléaire mais aussi, dans un contexte de crise pétrolière, de tensions croissantes entre l'entreprise publique et l'État. Après avoir opté pour la modernisation du service public, qui passe par une contractualisation accrue et la fixation d'objectifs pluriannuels, le ministère de l'Industrie, tutelle d'EDF, s'engage au milieu des années 1980 dans une nouvelle voie, celle de la mise en concurrence.

En 1986, le retour aux affaires d'une coalition de droite donne lieu à une première tentative d'application des nouvelles thèses économiques qui se font jour dans le secteur, et qui entendent réduire le monopole à sa portion congrue. Elle est l'œuvre d'Alain Madelin, qui hérite du portefeuille de l'Industrie sous le gouvernement formé autour du nouveau Premier ministre Jacques Chirac. Souhaitant s'inspirer de l'expérience britannique menée par Margaret Thatcher, Alain Madelin confie à Christian Stoffaes, économiste et polytechnicien du corps des Mines, le soin de rédiger un rapport sur les possibilités de déréglementation en France. La réforme a pour finalité de faire baisser les tarifs de l'électricité des industriels, dont le ministre estime qu'ils sont pénalisés par le système de subventions croisées mis en place par EDF depuis l'application de la tarification au coût marginal. Afin de remplir cet objectif, le ministère de l'Industrie entame une réflexion approfondie sur une série de mesures allant dans le sens d'une déréglementation partielle du secteur énergétique, comme l'introduction d'une dose de concurrence dans la production d'électricité ou le démantèlement de l'activité de distribution-fourniture d'EDF en entités régionales, à l'instar du modèle britannique. Dans la stratégie de changement envisagée par les réformateurs, les concessions communales de distribution-fourniture d'électricité qui ont été maintenues après la nationalisation sont appelées à jouer un rôle nouveau et important puisqu'il est prévu de les mettre aux enchères, sous le contrôle de l'État, afin d'accélérer le démantèlement du monopole public.

Si les réformes envisagées par Alain Madelin et son cabinet ne sont qu'un feu de paille qui se consume sans provoquer l'incendie que craignent alors les dirigeants d'EDF, elles n'en inaugurent pas moins une phase de profonde incertitude quant au devenir du secteur électrique français, et en particulier des concessions communales. À peine l'épisode Madelin refermé, une nouvelle étape s'ouvre avec la relance du processus d'intégration communautaire qui fait suite à la signature de l'Acte unique européen, en décembre 1985 (Schmidt, 1997, 1998; Levi-Faur, 1999). Après avoir été tentée par la mise en place d'un modèle fondé sur une intensification des échanges entre monopoles nationaux, la Commission européenne prône en 1988 une politique de libéralisation, influencée par la DG IV qui est chargée des questions de concurrence. Désormais, l'heure n'est plus à conserver tels quels les opérateurs existants, 
dans une logique de coopération interétatique. Il s'agit bel et bien de toucher à l'organisation même du secteur électrique et de donner la possibilité aux consommateurs, industriels puis particuliers, de pouvoir choisir librement leur fournisseur. Bien que les stratégies diffèrent entre réformateurs, entre partisans d'un changement radical à la britannique et défenseurs d'une évolution plus lente et graduelle, les projets ont ceci de commun qu'ils conduisent tous à déstabiliser le secteur de la distribution-fourniture et, partant, à susciter, en France, des interrogations sur le rôle et l'évolution du régime de la concession issu de la loi de 1906.

Ce nouveau contexte change considérablement la donne pour EDF. À l'instar de la GrandeBretagne, où la réforme menée par Margaret Thatcher a abouti à faire éclater la distributionfourniture et la confier à différentes entités, la France peut craindre une refonte majeure de cette branche d'activité, qui est pour l'essentiel aux mains d'EDF ${ }^{6}$. En la matière, l'entreprise publique n'est pas protégée par l'État, comme elle peut l'être sur le réseau de transport. Dans la distribution-fourniture, ce sont les collectivités locales qui sont formellement les maîtresses du jeu institutionnel, de par les compétences qui leur ont été conférées par la loi du 15 juin 1906, et qui n'ont jamais été abrogées après 1946. En remettant à plat le modèle français, la constitution d'un marché unique de l'énergie à l'échelle européenne met au jour une situation paradoxale et contestable au regard de la jurisprudence, qui n'avait jamais été remise en question auparavant : les communes peuvent contracter... mais avec un concessionnaire unique. En France, ce ne sont pas moins de 14000 contrats de concession qui sont concernés par l'entreprise de clarification induite par la nouvelle donne, des contrats qui sont aux mains de communes isolées ou, plus généralement, de syndicats intercommunaux organisés à l'échelle cantonale, voire plus rarement départementale.

\subsection{La distribution-fourniture d'électricité dans la décentralisation politico-administrative : retour vers un service urbain?}

Cette menace de démantèlement de la distribution-fourniture via les contrats de concession est d'autant plus crédible qu'elle intervient dans un contexte de décentralisation qui renforce considérablement la capacité d'action des collectivités locales (Poupeau, 1999). En théorie, la mise en concurrence de la distribution-fourniture pourrait se faire suivant le modèle britannique, qui ne donne aucun rôle aux collectivités locales. L'État pourrait envisager de supprimer le modèle de la concession, au motif qu'il est obsolète et caduc, et le remplacer par un système de licences : le territoire serait divisé en grandes entités et chaque opérateur choisi se verrait attribuer un monopole de distribution-fourniture assorti d'un transfert des actifs détenus par les collectivités locales. Dès lors, les communes et syndicats de communes n'auraient plus aucune fonction d'autorité concédante. Cette éventualité n'est à vrai dire guère envisageable au plan politique, au moment où la décentralisation vient d'être relancée par les lois de 1982 et 1983. La France est alors en pleine ébullition, avec l'émergence de ce qu'on appelle des « maires entrepreneurs » ou « managers » soucieux d'exercer pleinement leurs nouvelles prérogatives (Lorrain, 1991; Lorrain, 1993). En renforçant les compétences des communes en matière d'aménagement et d'urbanisme, de logement, de gestion de la voirie ou de développement local, la nouvelle donne institutionnelle érige les collectivités

\footnotetext{
${ }^{6}$ Notons toutefois la présence des entreprises locales de distribution, qui représentent $5 \%$ de la consommation totale d'électricité en France à cette époque. Sur ce point, cf Gabillet, 2015.
} 
locales en partenaires incontournables, des partenaires qui mobilisent de plus en plus l'outil de la délégation du service public pour assurer la gestion de l'eau, de l'assainissement, du transport public ou d'autres fournitures essentielles.

Bien qu'il demeure encore sous la tutelle de l'État et de ses deux monopoles publics, EDF et GDF, le domaine de l'énergie fait l'objet lui aussi d'une attention accrue de la part des collectivités locales. Certaines d'entre elles commencent à s'y investir plus fortement, via des opérations de maîtrise des consommations sur leur patrimoine ou en développant des réseaux de chaleur pouvant concurrencer l'électricité et le gaz (Poupeau, 1999; Bouvier, 2005 ; Lenoir, 2007). Mais surtout, en dotant les collectivités locales d'une plus grande liberté contractuelle (Auby, 1995), la loi du 2 mars 1982 menace de remettre en question les équilibres institutionnels d'après-guerre, autour de l'exercice du pouvoir concédant. Jusque-là, les communes ou intercommunalités autorités concédantes de la distribution-fourniture d'électricité n'avaient pas la faculté de négocier leur propre cahier des charges. La loi du 15 juin 1906 et le code général des collectivités locales les obligeaient à se conformer à un document type, fruit d'une négociation entre les représentants des collectivités locales et ceux de l'État, même s'il pouvait comporter des «blancs » permettant d'adapter les clauses générales aux spécificités territoriales. C'est sur cette ressource que les dirigeants d'EDF et les administrations d'État avaient longtemps joué pour faire avorter toute tentative de contractualisation de grande ampleur, quelques avenants signés dans les années 1960 ou 1970 permettant de répondre aux situations les plus sensibles (Poupeau, 2015a).

Avec les lois de décentralisation, qui desserrent l'étau sur les actes des collectivités locales, les communes ou syndicats de communes peuvent désormais, en théorie, contractualiser sans se référer à un cahier des charges type, élaboré au plan national. Tout au plus peut-on leur proposer un modèle qui n'a aucune valeur prescriptive (Auby, 1995). Ce faisant, la décentralisation introduit un élément de différenciation potentielle entre territoires, notamment en faveur des villes. Un maire pourrait profiter de l'arrivée à terme de son contrat de concession pour tenter de mieux l'adapter aux besoins spécifiques de sa commune. Il pourrait également arguer de certaines dispositions prévues dans les anciens cahiers des charges, et que ne respectent pas les centres de distribution, pour exiger l'ouverture de négociations rapides. En 1986, 31 contrats de concession arrivent à terme, 14 en 1987, 19 en 1988, 21 en 19897 . Certes, ces nouvelles marges de manœuvre restent encore virtuelles au tout début des années 1980. Les réflexions sur la décentralisation n'ont guère touché le secteur énergétique, qui est resté le pré carré des grands monopoles et du ministère de l'Industrie. L'option d'une régionalisation, qui avait été évoquée dans le programme de François Mitterrand, est rapidement écartée, de même que l'introduction d'une compétence de planification locale des réseaux (Bourjol, Le Lamer, 1984). En cette période de retour de la gauche au pouvoir, les socialistes et leurs alliés communistes ne veulent pas toucher au monopole d'EDF. Il faut donc attendre l'amorce d'une politique de libéralisation pour que la menace d'une mise en concurrence de la distribution-fourniture d'électricité redevienne crédible, ouvrant dès lors une période d'incertitude et d'interrogations multiples.

7 Source: «EDF et les collectivités locales», rapport du Comité de la Prospective, Direction Générale d'EDF, 22 mars 1990, page 17. 


\section{2. la mise sur l'agenda décisionnel d'une contractualisation}

C'est dans ce nouveau contexte que l'idée de négocier de nouveaux contrats de concession fait son chemin. Elle est portée par deux principaux acteurs, qui perçoivent assez rapidement la nécessité d'anticiper les conséquences d'un processus - la libéralisation - qu'ils considèrent comme inéluctable. Les dirigeants d'EDF, tout d'abord, souhaitent se prémunir contre tout risque de déstabilisation rapide et éviter un scénario à la britannique. Les élus ruraux, ensuite, regroupés au sein d'une vieille association créée en 1933, la Fédération nationale des collectivités concédantes et régies (FNCCR), craignent que les évolutions à venir ne profitent surtout aux grandes villes. Alors que leurs liens historiques avaient quelque peu tendance à se distendre depuis les années 1970, ces deux acteurs se rejoignent désormais dans une volonté commune : maintenir un modèle de service public centralisé.

\subsection{Entre menace et opportunité : les élus ruraux face aux changements institutionnels}

Dans une lettre datée du 21 novembre 1989, Pierre Delaporte reçoit, en sa qualité de PDG d'EDF, une demande officielle de Robert Morlevat, alors président de la FNCCR. Ce dernier l'invite à renégocier les contrats de concession avec les collectivités locales « pour conforter la pérennité d'EDF dans le futur marché européen de la distribution d'électricité et dans le contexte des nouvelles libertés communales ${ }^{8} »$. Il s'agit, toujours selon Robert Morlevat, de reconduire les contrats de concession « avant que ne s'ouvrent les inévitables discussions sur de nouvelles règles européennes pour les monopoles dans le domaine de l'énergie ${ }^{9} »$.

Cet appel émane d'une Fédération qui est bien connue de l'entreprise publique (Poupeau, 2015a). Née en 1933, la FNCCR a été en effet l'un des artisans de la nationalisation, qu'elle voit comme l'un des moyens de rééquilibrer les relations entre les communes rurales - qu'elle représente en majorité - et des concessionnaires privilégiant les zones les plus rentables, au premier rang desquelles figurent les villes. Cette position de soutien à la création d'un grand monopole public vaut à la FNCCR d'occuper une place centrale dans les nouvelles formes de régulation qui se mettent en place au sortir de la guerre. Les nombreuses relations qui se nouent entre elle et EDF autour de la tarification ou de l'électrification rurale dessinent une forme d'influence de type néo-corporatiste, où les arrangements informels, parfois noués dans une grande opacité, se substituent à la relation contractuelle d'avantguerre, formalisée à travers la signature d'un cahier des charges. En contrepartie de son soutien à EDF, la FNCCR dispose d'un quasi-monopole de représentation des intérêts des élus locaux, l'Association des maires de France lui déléguant de manière tacite la gestion des questions afférentes à l'énergie.

Lorsque se dessine la perspective d'une mise en concurrence d'EDF, c'est tout ce système d'échanges qui menace d'être mis à mal. Mais, dans le même temps, le contexte est porteur d'une formidable opportunité pour les élus ruraux ainsi que pour la Fédération qui les regroupe : revivifier une instance représentative en déclin depuis les années 1970. En effet, les adhérents de la FNCCR, à savoir, en majorité, les syndicats d'électrification organisés par canton ou par

\footnotetext{
${ }^{8}$ Lettre de Robert Morlevat, président de la FNCCR, datée du 21 novembre 1989 (source : «EDF et les collectivités locales », opus cité, page 144).

${ }^{9}$ Document joint à la lettre (ibidem).
} 
département, sont pour le moins moribonds au moment même où se dessinent les premiers projets de mise en concurrence. La Fédération a perdu de sa superbe face à EDF et à l'État, qui ont fortement réduit ses marges de manœuvre depuis la grande réforme tarifaire de 1963 (Poupeau, 2015a). L'exemple du Syndicat des communes de la banlieue parisienne pour l'électricité (SCBPE), l'une des structures intercommunales les plus importantes de France en matière d'énergie, illustre bien ce qu'il faut appeler une certaine apathie (Bellanger, Poupeau, 2013). Le SCBPE, qui est né en 1924, regroupe l'ensemble des communes de l'ex département de la Seine, soit 80 membres. Au début des années 1990, il gère un budget d'environ 120 millions de francs (soit environ 25 millions d'euros de 2014), composés de redevances diverses et de crédits d'électrification destinés aux communes. Mais sa capacité d'action est pour ainsi dire insignifiante. Sa structure administrative n'est guère étoffée et est mutualisée avec d'autres structures intercommunales de la région parisienne (eau, gaz, pompes funèbres). Le syndicat apparaît surtout comme un « gestionnaire de crédits » par lequel les ressources ne font que transiter car directement affectées, dans leur grande majorité, aux communes membres. Autrefois très présents et actifs, les maires des villes adhérentes sont de plus en plus en retrait de ses instances délibératives, ce qui atteste d'un manque d'attractivité réel du SCBPE. L'absentéisme des délégués sévit lors des réunions du bureau et du comité d'administration. Rédigé en 1990, un rapport de la Cour des comptes dénonce une structure qu'elle considère comme obsolète, un « intermédiaire administratif » sans réel pouvoir.

Ce constat d'un certain délitement des structures d'électrification héritées de l'entre-deuxguerres ne vaut pas que pour la banlieue parisienne. Il est manifeste dans beaucoup de syndicats de province, qui apparaissent très souvent comme des organismes délaissés par les élus locaux, lesquels leur préfèrent d'autres structures intercommunales plus attractives en termes d'enjeux politiques. Aussi la perspective d'une libéralisation apparaît-elle comme porteuse d'une vraie opportunité pour la FNCCR et sa base militante : se réaffirmer dans le paysage institutionnel local. L'évolution des prises de position de la Fédération en témoigne. Au début des années 1980, dès la parution des premiers textes de décentralisation, la FNCCR tient encore un discours assez défensif, qui consiste à modérer les ardeurs des élus. Elle ne plaide pas alors pour renégocier de nouveaux contrats de concession avec EDF. Tout au plus s'attache-t-elle à réaffirmer le caractère unitaire et solidaire du service public de l'électricité, qui passe, selon elle, par le maintien d'un cahier des charges national ${ }^{10}$. Mais, au fur et à mesure que les choses évoluent, les dirigeants de la FNCCR passent à l'offensive. Le statu quo ne leur paraît guère plus envisageable et c'est animé par ce sentiment de reconquête que Robert Morlevat écrit en 1989 à Pierre Delaporte, PDG d'EDF, pour l'inviter à engager le grand chantier de la négociation contractuelle que l'entreprise publique n'avait jamais véritablement mené jusqu'alors.

\subsection{Ouvrir la « boîte de Pandore » du « local » : la décision d'EDF de négocier de nou- veaux contrats de concession}

La demande exprimée par la FNCCR arrive à une période où les dirigeants d'EDF estiment que l'inaction n'est plus possible (Poupeau, 1999). Jusque-là, malgré une tentative avortée au début des années 1960 pour faciliter la mise en place de la tarification au coût

${ }^{10}$ Les services publics communaux et départementaux, bulletin d'informations de la FNCCR, $\mathrm{n}^{\circ} 145$, octobre 1982, page 23. 
marginal (Poupeau, 2015a), la négociation de nouveaux contrats de concession n'était pas considérée comme prioritaire. Bien au contraire, elle était plutôt perçue comme une source de contrainte supplémentaire pour l'entreprise publique : en revivifiant l'action des pouvoirs locaux, celle-ci ne risquait-elle pas de devoir gérer une double pression insupportable, entre des élus soucieux de défendre leurs usagers et leur territoire et un État jouant de sa position d'actionnaire et de tutelle pour imposer des décisions allant dans le sens de ses priorités nationales? Pour les dirigeants d'EDF, comme pour l'ensemble des agents de l'entreprise, il s'agissait non pas de nier les communes et leurs groupements mais de circonscrire leur action, dans un contexte de centralisation et d'étatisation accrues.

L'amorce d'un processus d'ouverture à la concurrence, second événement majeur de la décennie 1980 avec la décentralisation, remet en question ce point de vue, jusque-là solidement ancré dans la culture de l'entreprise publique (Poupeau, 1999). Bien que les intentions de la Commission européenne ne soient pas entièrement connues à la fin des années 1980, l'attirance des réformateurs de la DG IV pour une expérience radicale à la britannique fait peser une menace réelle et immédiate sur EDF. Les instances communautaires pourraient jouer sur le vide juridique créé par l'absence de contrats de concession signés en bonne et due forme pour accélérer l'éclatement du quasi-monopole ${ }^{11}$ dont l'entreprise jouit dans la distribution-fourniture, en imposant par exemple une mise en concurrence des concessions communales. Jean Bergougnoux, nouvellement nommé directeur général après le départ de Jean Guilhamon (et celui de Marcel Boiteux), en a bien conscience et joue un rôle décisif dans la conversion des services internes à l'idée d'une refondation institutionnelle. Peu à peu, les anciennes réticences s'estompent, du côté notamment des responsables de la Direction des services financiers et juridiques (DSFJ) ou de la Direction des études, de la prospective et de la stratégie (DEPS), qui voient dans le processus de refondation le moyen non plus d'affaiblir mais de protéger les bases de l'entreprise publique. Tout d'abord au plan juridique, dans la mesure où des contrats de concession actualisés seront plus difficilement attaquables et seront créateurs d'irréversibilités, donc d'inflexion par rapport au processus de libéralisation ${ }^{12}$. Ensuite au plan politique, dans la mesure où les élus locaux, qui trouveront de nouvelles marges de manœuvre à travers la négociation des cahiers des charges, pourront devenir des alliés précieux pour contrer l'action de la Commission. Enfin au plan commercial et managérial, la place accordée aux acteurs locaux rentrant parfaitement bien dans le cadre de la stratégie de l'entreprise, qui vise à modifier en profondeur le comportement des agents de la Direction de la distribution par le développement d'une culture de service et d'une politique de diversification (réseaux câblés et télésurveillance...). Solution adaptée à plusieurs types de problèmes qui se posent sur différents plans, la « refondation » n'apparaît alors plus comme inacceptable par les instances dirigeantes de l'entreprise.

Pierre Delaporte et Jean Bergougnoux décident d'accepter la proposition de la FNCCR et entament une phase de concertation en interne afin de dégager les grands axes d'une politique d'EDF en la matière. Un groupe de travail est constitué début 1990 et se penche sur le thème des relations avec les collectivités locales, sous l'angle institutionnel. Placé

\footnotetext{
11 Rappelons en effet l'existence des régies et SICAE, déjà évoquée plus haut.

12 Sauf à admettre la suppression pure et simple du régime de la concession de service public, option qui ne semble pas envisageable.
} 
sous la responsabilité du secrétaire du comité de gestion stratégique de l'entreprise, le groupe comprend sept autres personnes, issues des directions de la distribution (DD), des services financiers et juridiques (DSFJ), du développement et de la stratégie commerciale (DDSC), de la production et du transport (DPT), de l'économie, de la prospective et de la stratégie (DEPS, deux agents). Un auditeur de la Cour des Comptes complète l'équipe. Par les décisions stratégiques qu'il prend, par les verrous qu'il instaure préalablement à toute discussion avec les collectivités locales, ce groupe trace assez largement le chemin à prendre en vue de la refonte du cadre contractuel.

De janvier à mars 1990, les membres du groupe se réunissent afin d'élaborer une doctrine d'ensemble qui soit cohérente et qui puisse servir de canevas général aux futures négociations. L'ambiance est à l'émulation, dans la mesure où toutes les hypothèses d'avenir sont abordées, sans exclusive, y compris les cas de figure les plus subversifs par rapport à la situation de l'entreprise publique. Deux variables principales sont retenues, qui montrent bien l'état d'esprit dans lequel est pensé le processus de refondation. La première est le devenir du cadre réglementaire de la distribution-fourniture d'électricité en France, en particulier le maintien ou la remise en cause du monopole qui prévaut dans la gestion de cette activité. À l'époque, les réflexions qui s'engagent, de nature prospective, ne peuvent encore s'appuyer sur un cadre institutionnel stable. La Commission européenne n'a pas encore achevé ses réflexions sur le schéma de mise en concurrence retenu, ce qui laisse subsister un certain flou sur l'avenir du régime de la concession ${ }^{13}$. Eu égard à ces incertitudes, le groupe estime que « la question portant sur le point de savoir s'il convient de renouveler ou non les concessions doit donc être appréciée plutôt en fonction de critères d'opportunité qu'en termes juridiques, en l'état actuel du droit ${ }^{14}$ ».

La deuxième variable concerne le «niveau d'activité des prédateurs potentiels que sont, de concert ou non, les collectivités locales d'une part et les sociétés de distribution de services publics françaises ou étrangères d'autre part ${ }^{15}$ ». La sémantique utilisée (terme de prédation) montre bien que le problème des collectivités locales est alors formulé de manière « défensive », puisqu'il s'agit de contrer les éventuelles velléités de reprise en main de la distribution-fourniture par les acteurs locaux - en particulier les villes - aidés ou non des grands groupes de services. En fonction de ces deux variables, quatre scénarii sont élaborés ${ }^{16}$ :

\footnotetext{
${ }^{13}$ La Cour de justice des communautés européennes n'a pas encore élaboré de jurisprudence importante sur la question des concessions de service public. Seul l'arrêt « Bodson et SA Pompes funèbres des régions libérées » du 4 mai 1988 a soulevé le fait que l'existence d'un monopole territorial pouvait aller à l'encontre des échanges communautaires («EDF et les collectivités locales », opus cité, page 71).

${ }^{14} \mathrm{lbidem}$, page 69.

${ }^{15} \mathrm{Ibidem}$, page 76.

${ }^{16}$ Le schéma qui suit reprend de façon synthétique les conclusions du groupe de travail (source : «EDF et les collectivités locales », opus cité).
} 
Figure 1 : Les quatre scénarios envisagés par le groupe de travail EDF collectivités locales

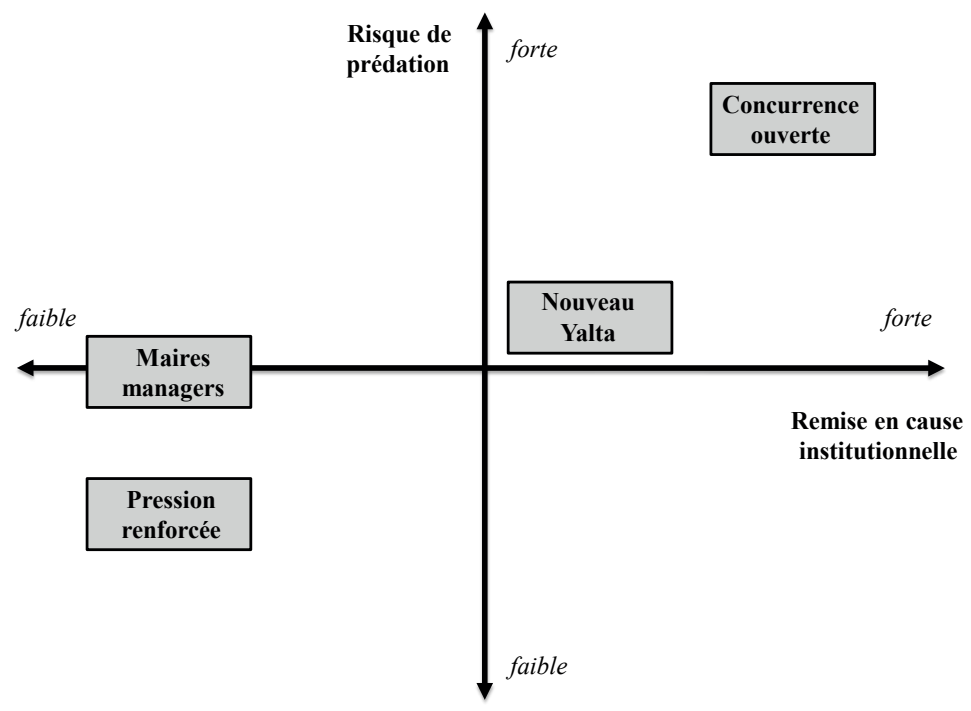

Dans les cas de «pression renforcée dans la continuité » et de «maires-managers », le cadre institutionnel est globalement maintenu. Il n'y a pas transformation majeure du secteur de la distribution-fourniture ni mise en concurrence d'EDF sur les concessions. Seule diffère la volonté des collectivités locales de s'investir dans le domaine de l'énergie, par l'extension de quelques régies urbaines, par un contrôle accru des cahiers des charges ou par la soumission plus grande du concessionnaire EDF à des exigences locales (coordination des travaux...). Le scénario du « nouveau Yalta » est celui que le groupe de travail souhaite le moins, dans la mesure où il correspond à un grignotage diffus et progressif des positions d'EDF, sans remise en cause globale. Dans cette hypothèse, EDF se voit amputée, au profit des collectivités locales et des grands groupes, de fonctions situées en aval de la distribution-fourniture et qu'elle remplit habituellement : contact avec la clientèle, opérations de relève, de facturation, d'encaissement, petites interventions sur le réseau. Ces fonctions, qui sont assurées par des services communs à différentes activités de délégation de service public, sous la supervision des collectivités locales, font émerger une gestion locale multiservices (électricité, gaz, eau...). Il conduit, selon le groupe de travail, à démembrer en partie EDF, puisque 15000 personnes pourraient être alors touchées. Le dernier scénario, « concurrence ouverte », part de l'hypothèse d'un bouleversement du cadre institutionnel par une mise en concurrence des concessions, avec la volonté des collectivités locales de jouer un rôle-clé dans la réorganisation de la distribution-fourniture, grâce au soutien actif des grands groupes.

Eu égard à ces quatre scénarii, des stratégies de riposte sont élaborées. Elles visent à répondre à des scénarii de type « pression renforcée dans la continuité », « maires-managers » (les deux scénarii qui ont la préférence du groupe) et « concurrence ouverte », en évitant surtout qu'un « nouveau Yalta » n'émerge. Dans tous les cas de figure retenus, la négociation de nouveaux contrats de concession apparaît indispensable aux membres du groupe, tant 
elle semble au cœur des évolutions à venir du système de distribution-fourniture, quelle qu'en soit l'issue. Le groupe s'efforce surtout de réfléchir aux conditions pouvant produire l'émergence des deux scénarii qui ont leur faveur, ceux qui ne conduiraient pas à un fort bouleversement du modèle d'entreprise, préparant des parades éventuelles si d'aventure les autres cas de figure se dessinaient dans un avenir plus ou moins proche.

\section{3. le contrat contre le marché}

C'est donc sur ces bases stratégiques qu'EDF entame les négociations contractuelles. D'emblée, l'entreprise décide d'ériger la FNCCR en interlocuteur exclusif pour établir un nouveau modèle de cahier des charges. L'enjeu est de taille pour les deux partenaires : il s'agit d'orienter l'ensemble des communes et syndicats de communes ayant compétence d'autorité concédante vers l'option retenue par les deux protagonistes, à savoir le maintien d'un système centralisé dans lequel les villes ne seront pas tentées de s'émanciper. Le succès de ce chantier passe par la rédaction d'un cahier des charges outillant les négociateurs locaux pour leur permettre d' « enrôler » les élus autour de ce «script » pré-établi (Akrich, 1987). L'élaboration de ce document constitue le cœur même des négociations qui s'ouvrent entre EDF et la FNCCR au tournant des années 1980 et 1990.

\subsection{L'alliance avec le monde rural}

Suite à la rédaction du rapport sur les collectivités locales, des discussions s'engagent au sein du comité de gestion stratégique d'EDF afin de fixer le cadre général des pourparlers avec les autorités concédantes. L'idée d'une contractualisation fait l'objet d'un large consensus, les participants considérant qu'une telle refonte contribue à maintenir le système en l'état, à court et moyen termes. Cependant, ce «nécessaire » chantier contractuel ne saurait s'accompagner d'une redistribution trop importante des pouvoirs en direction des collectivités locales. Face à un État qui ne semble pas prêt à abandonner ses prérogatives de tutelle et d'actionnaire, les membres du comité craignent qu'une renaissance trop brusque des autorités concédantes ne limite encore plus les marges de manœuvre de leur entreprise et qu'elle ne grève trop lourdement ses résultats. Aussi, alors qu'il semblait possible au plan théorique, le choix d'une alliance forte avec les communes contre l'État est-il rejeté, EDF s'inscrivant toujours dans la perspective d'un système de régulation fortement encadré par les ministères des Finances et de l'Industrie. La volonté de se protéger de la libéralisation, le souci de ne pas créer un second contrepouvoir aux côtés de l'État incitent le groupe de travail à opérer trois choix stratégiques majeurs (Poupeau, 1999) : le maintien d'une péréquation nationale des tarifs, l'alliance avec le monde rural (et donc la FNCCR), le souci d'une redistribution limitée de la rente générée par l'activité de distribution-fourniture.

À un moment où il n'est pas encore question de séparer formellement « acheminement » et «fourniture » de l'énergie électrique (unbundling), la question tarifaire constitue un élément central du processus de refondation institutionnelle : faut-il laisser les collectivités locales négocier, en leur qualité d'autorités concédantes, des tarifs différenciés selon les territoires? faut-il, ce faisant, aller dans le sens d'une 
différenciation croissante dans le traitement des usagers? Ces questions relatives aux tarifs renvoient d'emblée au maintien ou non de la péréquation géographique des tarifs acquise durant les années 1960 et 1970 (Poupeau, 2007). Dans la mesure où elle n'est pas inscrite dans la loi, la péréquation peut être défaite par une décision relevant d'un choix de l'entreprise, de l'État et/ou des collectivités locales, sans pour autant remettre en cause la nationalisation. Pour prendre un cas limite, il serait en théorie envisageable qu'un maire de grande ville décide de négocier son propre cahier des charges et fasse pression pour que les habitants de sa commune bénéficient de tarifs spécifiques ${ }^{17}$. De son côté, l'entreprise publique, se projetant dans un avenir où elle peut estimer que les marchés seront de plus en plus concurrentiels et donc soumis à une logique de différenciation, y compris tarifaire ${ }^{18}$, pourrait être tentée de pousser les pouvoirs publics à une discrimination tarifaire. Sur cette question, la position du groupe de travail est claire : il faut maintenir telle quelle la péréquation afin d'éviter un éclatement de l'activité de distribution-fourniture, les villes pouvant jouer sinon de cette latitude d'action pour s'émanciper aux côtés de nouveaux entrants, les groupes de services. Afin d'étayer et de conforter cette position, des enquêtes sont réalisées par l'entreprise auprès de la population française. Il en résulte un fort degré d'attachement à ce qui est considéré comme un principe fondamental du modèle français de service public. D'autres études montrent également l'importance que la péréquation revêt aux yeux des responsables politiques nationaux et locaux, malgré les différences idéologiques qui partagent la classe politique française quant à la gestion du service public. Enfin, au plan interne, la prégnance de la CGT et d'un modèle égalitariste de service public confortent la décision du groupe de maintenir des tarifs uniformes sur l'ensemble du territoire.

Le choix des interlocuteurs à privilégier découle de cette stratégie de containment. Lorsqu'EDF se penche sur le dossier des concessions, il existe encore 14000 autorités concédantes sur le territoire national. Si l'idée de favoriser des regroupements de communes est d'emblée admise par les négociateurs d'EDF, la question de la « maille concessive » reste pleine et entière. Jusqu'où faut-il réduire le nombre d'interlocuteurs? faut-il privilégier un partenaire aux plans national (définition du cahier des charges modèle) et local (négociation des contrats de concession)? faut-il jouer la carte du pluralisme et inviter, aux côtés de la FNCCR, les autres associations d'élus à négocier le nouveau cahier des charges, notamment celles qui représentent les intérêts des villes, grandes (Association des maires des grandes villes de France, AMGVF) et moyennes (Fédération des maires des villes moyennes, FMVM)? Là encore, la réponse à cette série de questions est loin d'être neutre au plan institutionnel. Très rapidement, le groupe de travail interne à EDF en vient à préconiser d'inciter les communes à se regrouper à une maille intercommunale assez large, sur la base notamment des syndicats d'électrification rurale existants. Deux raisons militent à ses yeux pour une telle stratégie de regroupement. La première est de tenter de neutraliser les villes en les incluant dans de vastes ensembles territoriaux où les intérêts du monde rural

\footnotetext{
${ }^{17}$ Même s'il convient de nuancer cette hypothèse, qui se veut extrême. En effet, malgré l'abrogation en 1986 de l'ordonnance de 1945 sur le contrôle des prix par l'État, le ministère des Finances conserve toujours la maîtrise des tarifs des services publics.

${ }^{18} \mathrm{Ne}$ serait-ce, par exemple, que dans l'optique d'une concurrence entre énergies elles-mêmes non péréquées, comme c'est le cas pour le gaz, le fioul, le propane. Ces énergies pourraient pénétrer certains marchés urbains plus facilement que l'électricité.
} 
seront fortement représentés. La seconde est de contrer les éventuelles stratégies d'entrisme des grands groupes de services et de les empêcher de ne racheter que les concessions les plus juteuses sur le plan de la rentabilité. En constituant de vastes concessions, qui mêlent urbain et rural et qui représentent des sommes importantes à débourser ${ }^{19}$, EDF entend ainsi décourager ses concurrents. Pour autant, elle reste réticente à aller vers une maille purement départementale, de crainte d'avoir des interlocuteurs - les syndicats d'électrification - trop puissants en face d'elle. Eu égard à ces considérations stratégiques, l'entreprise décide de renouveler son alliance historique avec le monde rural et, plus précisément, avec la FNCCR. Fédération fortement liée à EDF car tirant profit de la centralisation du secteur électrique, la FNCCR, même affaiblie, est alors considérée comme un allié précieux pour l'entreprise publique, susceptible de mobiliser les élus locaux autour de la stratégie de maintien de la centralisation prônée par les « refondateurs » (Poupeau, 1999).

Le problème du partage avec les collectivités locales de la rente générée par l'activité de distribution-fourniture constitue le dernier élément principal de la stratégie d'EDF. Là encore, plusieurs schémas sont possibles. À un extrême, on peut envisager un relatif statu quo par une redistribution minimale, l'essentiel de la rente étant toujours accaparé par l'entreprise publique, pour son autofinancement, et par l'État-actionnaire. À l'autre extrême, à une période où EDF s'engage dans des actions de diversification vers des marchés où les collectivités locales jouent un rôle décisionnel prépondérant, le choix peut être fait d'adopter une attitude plus généreuse. Une stratégie d'alliance très forte avec les autorités concédantes serait envisageable pour EDF. Selon les cas de figure, on peut en effet imaginer une dépense supplémentaire de l'ordre de 2,5 milliards de francs (plus de $500 \mathrm{~m} €$ de 2014) pour l'activité de distribution-fourniture, dont 1,7 milliard de francs (un peu moins de $400 \mathrm{~m} €$ de 2014) de redevances versées aux collectivités locales $^{20}$. C'est sur cette question que les avis sont les plus partagés, révélant des différences d'appréciation importantes entre les acteurs. Ceux qui, dans une optique essentiellement tacticienne, se sont tardivement ralliés à la stratégie de refondation ou qui restent attachés à une faible redistribution des pouvoirs, défendent avec vigueur la thèse d'un montant minimal. Le comportement de l'État, qui a tendance à ponctionner lourdement l'entreprise sur ses bénéfices, milite, selon eux, pour réduire à un seuil minimal les transferts financiers vers les autorités concédantes. Si la stratégie de l'alliance forte avec les pouvoirs locaux ne rencontre guère de partisan convaincu, un autre camp défend l'idée que le partage de la rente doit être suffisamment important pour que les communes soient incitées à négocier rapidement leurs contrats de concession avec EDF. Une attitude trop timorée pourrait selon eux conduire à des conséquences graves et, pourquoi pas, à une déstabilisation du secteur électrique. Finalement, au terme d'un arbitrage de Jean Bergougnoux, sous la forte mais discrète pression du ministère des Finances, les premiers obtiennent gain de cause, le niveau des redevances versées aux autorités concédantes étant proche des hypothèses basses.

\footnotetext{
19 L'ensemble des actifs se chiffrant à plusieurs milliards de francs de l'époque (soit plus d'un milliard d'euros actuels), même si la question de leur reprise en fin de concession n'est pas encore réglée au plan juridique. ${ }^{20}$ Source : «EDF et les collectivités locales », opus cité, pages 37-38. Le reste correspondant notamment à un accroissement des investissements sur les réseaux de basse et moyenne tensions.
} 


\subsection{Faire du cahier des charges un document attractif : petit précis d'« ingénierie territoriale »}

Ayant fixé les principes cardinaux d'une refonte des liens contractuels avec les autorités concédantes, les dirigeants d'EDF peuvent se lancer dans la phase de négociation d'un nouveau cahier des charges. La FNCCR est leur seul interlocuteur. À l'automne 1990, un groupe de travail est formé afin de mettre en œuvre les orientations définies par le comité de gestion stratégique d'EDF. Il est composé de huit représentants, répartis à parité entre les deux protagonistes. Du côté d'EDF, on retrouve deux personnes de la DEPS et deux juristes. La tâche de contractualisation est confiée au chef du service tarification de la DEPS, qui représente la tendance plutôt « conservatrice » d'EDF. Avec lui, les risques de « dérapage » apparaissent donc minimisés. Pour la FNCCR, les négociations sont menées par l'un des vice-présidents de la Fédération, qui a également été son directeur dans les années 1970 et 1980, par le directeur en poste et deux chargés de mission qui changent suivant les sujets. Le groupe travaille de façon isolée pendant près d'un an, à raison de deux réunions par mois en moyenne. Aucun représentant de l'État n'est présent, aucun membre d'associations d'élus ou de fonctionnaires territoriaux non plus. Cette négociation à huis clos témoigne d'une volonté de maîtriser le processus de contractualisation et d'éviter que certaines catégories de collectivités locales, notamment les grandes villes, ne soient tentées de faire valoir à ce stade leurs intérêts particuliers.

Même si elle a, par son poids institutionnel, la main sur le processus, EDF doit faire cependant des concessions importantes à ses interlocuteurs de la FNCCR. Celles-ci portent sur deux points majeurs. Le premier a trait au périmètre sur lequel doit s'opérer le regroupement des communes. Si les deux partenaires sont d'accord pour réduire le nombre d'autorités concédantes, des différends demeurent sur la taille des futurs ensembles intercommunaux. EDF souhaite favoriser une maille suffisante mais sans nécessairement épouser les frontières départementales. Elle peut en effet craindre, nous l'avons dit, de voir se constituer des syndicats départementaux très puissants et pouvant, à terme, devenir de vrais contre-pouvoirs locaux. De son côté, la FNCCR pousse à une départementalisation, fidèle à la stratégie qu'elle a toujours poursuivie depuis sa création en 1933 (Poupeau, 2015a). Pour elle en effet, l'échelon départemental permet d'établir une solidarité solide entre villes et campagnes. Il correspond à un espace politique auquel ses adhérents, le plus souvent des sénateurs ou conseillers généraux, sont habitués. Il permet d'avoir des syndicats ayant suffisamment de poids pour peser sur les décisions du concessionnaire. Sur ce premier sujet, EDF est obligée de lâcher du lest, à travers, nous le verrons, des mécanismes incitatifs qui poussent clairement à la départementalisation.

Le second point de différend concerne l'adoption d'une « clause de la concession la plus favorisée ». Elle constitue un second élément majeur du dispositif mis en place pour « formater » l'espace institutionnel local. Elle stipule que toute mesure spécifique prise lors d'une négociation aura vocation à s'étendre aux autres contrats de concession, même s'ils ont déjà été signés. Là encore, EDF se montre réticente à accepter une clause qui ne peut que réduire ses marges de négociations, notamment avec les grandes villes. La FNCCR y est plus favorable, y voyant la garantie d'un traitement égalitaire de l'ensemble des autorités concédantes. Par ce biais, la Fédération entend ainsi faire bénéficier l'ensemble de ses adhérents des avantages acquis dans un endroit quelconque de l'Hexagone. Elle y voit également le moyen d'éviter que certaines communes plus importantes ne bénéficient de dispositions particulières auxquelles les communes rurales n'auraient pas droit. 
Avec ce mécanisme, le cahier des charges, qui ne pouvait être de jure qu'un « modèle », se mue de facto en un document « type », par construction d'une forme d'interdépendance subtile entre les scènes locales de la négociation contractuelle. Cette mécanique uniformisatrice est renforcée par la décision de mettre en place des structures de suivi et d'arbitrage. La FNCCR et EDF ont chacune leur cellule assurant le pilotage de la mise en œuvre du cahier des charges. À EDF, cette fonction est remplie par un juriste entouré d'une petite équipe, par lesquels doit passer toute décision à caractère problématique ou stratégique. Enfin, EDF et la FNCCR décident de se voir régulièrement pour faire le point sur les négociations et régler les litiges qui peuvent émerger localement. Ces différentes instances permettent d'assurer une forte harmonisation contractuelle.

Durant la petite année au cours de laquelle il se réunit, le groupe de travail s'efforce de traduire ces orientations stratégiques dans ce qui doit être le futur modèle de cahier des charges des concessions de distribution publique d'électricité. Il s'agit très concrètement de trouver et mettre en forme une solution à l'équation subtile et difficile qu'il est donné de résoudre entre nécessité de rendre le nouveau cahier des charges attractif (pour inciter les élus à contractualiser) et souci de limiter les marges de manœuvres locales (pour éviter toute forme de différenciation voire de surenchère entre communes). Il s'agit aussi, et plus particulièrement, de faire en sorte de transformer un document qui ne reste qu'indicatif (le cahier des charges modèle) en un instrument prescriptif (un cahier des charges type) à même de maintenir l'ensemble des collectivités locales dans un cadre contractuel garantissant une centralisation que les deux partenaires appellent de leurs vœux, quoiqu'avec des intérêts différents.

L'économie du contrat ne saurait en effet être bouleversée. Comme on l'a vu, les mécanismes de péréquation sont maintenus, que ce soit pour les tarifs ou pour les investissements. Dans ce cadre général où les déterminants principaux de l'activité de distribution-fourniture publique d'électricité restent fixés par le niveau central, en lien avec l'État, les espaces de négociation ne peuvent être que très limités. Le nouveau cahier des charges prévoit de mieux organiser le contrôle local des concessions, au plan patrimonial notamment (connaissance des réseaux, contrôle des comptes de concession via un compte-rendu annuel d'activité). Il comprend des dispositions relatives à la répartition de la maîtrise d'ouvrage entre EDF et les autorités concédantes (ce qui n'est pas sans importance car la maîtrise d'ouvrage détermine notamment l'origine de la décision d'investissement). Il prévoit enfin de laisser les parties prenantes négocier chaque année une enveloppe globale de financement de mesures en faveur de l'environnement : amélioration de l' « esthétique des réseaux », mise en technique discrète ou passage en souterrain (article 8)... Hormis ces quelques mesures, il est difficile de parler de véritable espace de négociation local, tant les dimensions nationales pèsent dans l'élaboration du cahier des charges.

Dans ce contexte, le principal levier mobilisé pour susciter l'intérêt et l'adhésion des élus locaux est de nature financière, grâce aux quelques centaines de millions de francs qu'EDF a décidé d'injecter dans la stratégie de contractualisation. Il s'agit de poursuivre un double objectif : inciter les élus à signer rapidement les nouveaux traités de concession tout en favorisant la constitution de vastes ensembles territoriaux regroupant les autorités concédantes. Pour ce faire, les membres du groupe de travail mettent au point deux redevances nouvelles. La première, dite « R1 » ou « redevance de fonctionnement », a pour but de permettre à l'autorité concédante d'exercer un contrôle du concessionnaire (embauche d'un ou plusieurs techniciens qualifiés, équipements 
éventuels...). Elle se substitue à la redevance de contrôle qui existait antérieurement. La seconde, dite $\mathrm{R} 2$ ou « redevance d'investissement », est destinée à compenser une partie des dépenses que les collectivités concédantes peuvent effectuer, en tant que maîtres d'ouvrage, sur les réseaux d'électricité. C'est en particulier le cas d'interventions ayant pour cadre l'électrification rurale ou certains travaux d'extension réalisés par des syndicats d'électrification ou des communes. Ce sont via ces deux redevances que les rédacteurs du cahier des charges escomptent inciter les communes à se regrouper. La formule de calcul retenue pour chacune d'entre elle, outre le fait qu'elle favorise une durée de contrat longue ${ }^{21}$, comprend un facteur multiplicateur en fonction de la population couverte par la concession : $1+\mathrm{P}_{C} / \mathrm{P}_{\mathrm{D}}\left(\mathrm{P}_{\mathrm{C}}\right.$ : population municipale de la concession; $\mathrm{P}_{\mathrm{D}}$ : population municipale desservie par EDF dans le département où se situe la concession). Ainsi, un syndicat totalisant $100 \%$ des habitants de son département bénéficiera d'un doublement de sa redevance, quand un autre, qui n'en couvre que $20 \%$, ne se verra appliquer qu'un facteur de 1,2. Par ce système très incitatif, EDF et la FNCCR cherchent donc à « enrôler » les élus locaux dans leur stratégie d' « ingénierie territoriale ». La tâche s'avère cependant ardue pour les « refondateurs », dans la mesure où, conséquence des politiques antérieures menées par EDF et les pouvoirs publics, il n'existe pas toujours de syndicat départemental structuré, y compris pour gérer les crédits d'électrification rurale. Une cinquantaine d'organisations tout au plus semblent pouvoir se targuer de jouer un tel rôle ${ }^{22}$, et encore sous réserve pour certaines d'entre elles de se voir transférer le statut d'autorités concédantes par les communes de leur territoire. Dans les autres départements, le pouvoir concédant est émietté et, la plupart du temps, détenu en majorité par les communes. Le risque d'échec de la stratégie d'EDF et de la FNCCR existe donc bel et bien.

\section{Du cahier des charges au contrat : contestations, adaptations, mise en œuvre}

Dotés d'un cahier des charges « modèle » qu'ils voudraient ériger de fait en document « type », les dirigeants d'EDF et de la FNCCR rendent public, courant 1991, le fruit de leurs pourparlers. D'emblée, des critiques s'élèvent dans les rangs des représentants des collectivités locales. Elles émanent de deux principales associations, l'une d'élus (Association des Maires des Grandes Villes de France, AMGVF), l'autre d'ingénieurs territoriaux (Association des Ingénieurs des Villes de France, AIVF). Malgré leurs protestations, ces dernières ne peuvent cependant faire front face à l'alliance solide qui a été nouée entre les deux partenaires historiques. Elles n'obtiennent que quelques compensations mineures et sont assez démunies pour s'opposer au processus de départementalisation progressive qui gagne la distribution-fourniture publique d'électricité dans les années 1990 et 2000.

\subsection{Faire face aux contestations : I'introduction de nouveaux espaces de négociations}

Après un an d'élaboration, le nouveau cahier des charges est étrenné, à l'automne 1991, à l'occasion du congrès de la FNCCR de Strasbourg, le premier tenu depuis 1982. Cette

\footnotetext{
${ }^{21}$ À travers l'intégration d'un terme " $\mathrm{D}$ », qui accorde une « prime » aux contrats longs, dans une limite que les négociateurs fixent à 30 ans maximum. Sur les formules exactes de calcul de R1 et de R2, voir l'annexe 2 . ${ }^{22}$ FNCCR, « Congrès de Strasbourg », 16-19 septembre 1991, FNCCR, 1991, page 55.
} 
manifestation constitue incontestablement une « résurrection » pour une Fédération ayant eu du mal à exister dans le paysage institutionnel depuis la fin des années 1970. Avec cette présentation officielle s'ouvre une phase de concertation avec les acteurs qui n'avaient pas été associés au cahier des charges. Elle s'étend de novembre 1991 à avril 1992, période durant laquelle des séances sont organisées sous l'égide à la fois des ministères de l'Industrie et de l'Intérieur. Si le monde des collectivités locales - associations d'élus locaux et de fonctionnaires territoriaux - conteste quelque peu les options prises, en revanche, les différentes administrations d'État soutiennent le projet tel qu'il a été adopté.

Assez rapidement, EDF et la FNCCR doivent faire face à la montée d'un courant protestataire au sein des collectivités locales. Sans surprise, il émane essentiellement des grandes villes, lesquelles trouvent que le modèle contractuel proposé correspond davantage aux intérêts des zones rurales. Ce sentiment est partagé par certains syndicats urbains membres de la FNCCR, comme le SCBPE, qui reproche au document qui lui est soumis de ne pas assez tenir compte de ses problématiques propres (Bellanger, Poupeau, 2013). En particulier, deux associations nationales, l'une d'élus, l'autre de fonctionnaires territoriaux, tentent de mener la rébellion (Poupeau, 1999). La première est l'AMGVF, qui souhaite différencier le traitement des grandes villes vis-à-vis des autres communes, revendication qui n'avait jamais été exprimée après-guerre, dans un système institutionnel où seule la FNCCR se voyait reconnue comme représentante des collectivités locales. L'officialisation du cahier des charges négocié par EDF et la FNCCR ouvre pour elle une voie nouvelle dans le domaine de la distribution-fourniture d'électricité. L'AMGVF se fait désormais force de pression vis-à-vis des pouvoirs publics (Palard, 1996), et non plus simple instance de professionnalisation des élus (Pétaux, 1994). Absente des pourparlers entre EDF et la FNCCR, elle rentre dans le champ des discussions au cours de la procédure de concertation durant laquelle les pouvoirs publics l'enjoignent à donner son avis sur le modèle de cahier des charges. Le 12 décembre 1991, un groupe de travail est constitué et placé sous la présidence de MM. Charreau et Piémontesi, respectivement adjoints au maire d'Angers et de Dijon ${ }^{23}$. Il est chargé d'évaluer les implications du nouveau cahier des charges pour les grandes villes, à un moment où la question de la délégation de services publics constitue un thème de réflexion important pour l'AMGVF (Pétaux, 1994). L'association défend quatre revendications majeures ${ }^{24}$. La première est que le cahier des charges ne saurait être qu'un modèle et non un document contraignant, ce qui doit laisser une large place aux négociations locales. La seconde concerne la maîtrise du développement des énergies sur le territoire communal : celle-ci ne saurait échapper au maire. La troisième revendication concerne le libre transit sur le réseau d'EDF, afin de permettre aux grandes villes d'alimenter certains de leurs services avec de l'électricité autoproduite par des usines d'incinération notamment. Enfin, l'AMGVF demande que le calcul du montant des redevances d'occupation du domaine public soit révisé, afin de combler les disparités entre villes, puisque Reims touche, à ce titre, cinq millions de francs (un million d'euros de 2014) lorsque la ville d'Angers ne perçoit que... 200 francs. Pour un syndicat comme le SCBPE, l'application stricte des clauses du cahier des charges entraînerait un manque

\footnotetext{
23 "Grandes Villes Hebdo », 4 décembre 1992, pages 3-4.

${ }^{24}$ La transformation du régime de la concession en affermage, réclamée par certains maires, n'est pas officiellement évoquée.
} 
à gagner de 50 à 100 millions de francs selon les estimations (11 à 22 millions d'euros de 2014), ce qui est jugé inacceptable (Bellanger, Poupeau, 2013).

Les contacts se multiplient alors avec les responsables d'EDF. Face aux réticences manifestées par ces derniers, la stratégie de l'AMGVF est de jouer la carte de l'attentisme (CNFPT, 1993). Afin de ne pas bloquer la situation à un moment où les négociations avec les instances communautaires rentrent dans une phase décisive, les dirigeants d'EDF acceptent de signer fin 1995 un «protocole grandes villes ». N'étant pas en soi un document contractuel - ce que ne manquent pas de signaler les élus concernés -, ce protocole offre une certaine flexibilité car il permet d'échapper à la « clause de la concession la plus favorisée » contenue dans le modèle de cahier des charges négocié avec la FNCCR. Le protocole offre peu d'avancées sur le fond, excepté sur un point majeur : les aspects financiers. Il permet en effet de maintenir le montant des anciennes redevances et d'ouvrir des espaces de négociation supplémentaires à travers des « conventions de partenariat». Dans beaucoup d'aires urbaines, la situation se débloque. Faute de disposer de leviers d'action supplémentaires, l'AMGVF doit se contenter de ces adaptations, certes importantes, mais qui ne remettent pas en cause les fondements de la stratégie de contractualisation co-élaborée par EDF et la FNCCR.

L'AIVF est l'autre force contestataire la plus active à l'égard du nouveau cahier des charges. Il faut dire que les ingénieurs des services techniques - que regroupe l'AIVF - sont alors, avec les élus locaux, les acteurs les plus concernés a priori par la « refondation ${ }^{25}$ ». En charge des questions techniques, ils constituent des interlocuteurs réguliers pour les agents des centres de distribution d'EDF-GDF. S'occupant également de la gestion des consommations d'énergie et du suivi des réseaux de chaleur, ils disposent d'une expertise qui peut être utile dans la négociation de nouveaux traités de concession. Or, ils sont d'emblée exclus des discussions portant sur le contenu du nouveau cahier des charges. Cette attitude d'EDF et de la FNCCR n'est pas totalement fortuite. À travers son « groupe énergie », l'AIVF promeut avec vigueur, depuis les années 1980, un modèle de gestion décentralisé, qui donnerait la possibilité aux communes de mener des politiques énergétiques locales (Poupeau, 1999). Certains de ses membres dirigeants ont d'ailleurs essayé d'innover en la matière, dans des villes comme Rennes, Montpellier ou Limoges. Aussi la stratégie de contournement de l'AIVF pratiquée par EDF et la FNCCR est-elle ressentie comme un véritable camouflet par beaucoup de directeurs des services techniques, et ce d'autant plus qu'elle se conjugue à une série d'évolutions locales qui tendent à diminuer le rôle qu'ils occupent, au profit des secrétaires généraux (Roubieu, 1994). L'attitude de l'AIVF est pour le moins très offensive. Dès la publication du nouveau cahier des charges, des colloques sont organisés, dont le plus important se tient à Montpellier à la fin de l'année $1992^{26}$. Rassemblant un grand nombre d'acteurs des collectivités locales, sous l'égide de l'AIVF et du Centre national de la fonction publique territoriale (CNFPT), il lance la « croisade »

\footnotetext{
${ }^{25}$ Pour ne pas alourdir la présentation, nous n'évoquerons pas le rôle des Secrétaires Généraux. En effet, ces derniers sont assez peu présents dans les négociations, comparativement aux élus locaux et aux directeurs des services techniques. Ils ont été toutefois associés aux réflexions de l'AMGVF. En outre, même s'il apparaît encore très réduit, il faudrait mentionner le rôle de l'Association des Administrateurs Territoriaux de France (AATF), créée en 1988, et qui organise son premier colloque en juin 1993 sur le thème de «l'avenir du service public local » (Roubieu, 1994).

${ }^{26}$ On peut trouver le verbatim des échanges dans l'ouvrage édité par le CNFPT (CNFPT, 1993).
} 
contre EDF et sa conception de la politique énergétique. Ce colloque est relayé par de multiples réunions locales, souvent soutenues par l'Agence de l'environnement et de la maîtrise de l'énergie (ADEME), et qui vont beaucoup plus loin que la simple économie concessionnaire, en défendant une politique énergétique beaucoup plus décentralisée. $\mathrm{Au}$ final, l'action de l'AIVF apparaît comme ayant une certaine portée car elle oblige à pousser la logique de différenciation jusqu'aux limites compatibles avec la stratégie intégratrice d'EDF et de la FNCCR. L'expertise de son « groupe énergie » permet à beaucoup d'ingénieurs des services techniques de décrypter les enjeux de la contractualisation. La force de son réseau facilite les échanges entre communes, les résultats obtenus dans une ville étant assez rapidement connus des autres collectivités, ce qui permet parfois de pousser EDF dans ses derniers retranchements.

\subsection{La lente départementalisation du paysage institutionnel énergétique local}

Dès 1992, avant même que ne soient réglés les différends avec l'AMGVF et l'AIVF, le processus de négociation locale des contrats de concession peut être entamé (Poupeau, 1999). Loin de se contenter des dispositifs de «formatage » prévus dans le cahier des charges, les dirigeants d'EDF et de la FNCCR suivent de près les négociations, pour éviter tout risque d'enlisement ou de dérapage. Afin d'initier une forte mobilisation locale, le président de la FNCCR préconise de commencer par certaines régions particulièrement bien préparées. En accord avec EDF sont choisis des départements dont la FNCCR sait qu'ils sauront fédérer la totalité des communes ou, à défaut, une très grande majorité d'entre elles. De ces premières réussites, on escompte impulser une dynamique de nature à entraîner les élus des autres départements. D'où, parallèlement, un certain atermoiement manifesté dans des centres EDF-GDF plus urbains, qui sont priés d'attendre les premiers résultats des autres négociations. Dans tout le pays, les responsables de la FNCCR mais aussi les directeurs de centres et les chefs d'agences de la direction de la distribution d'EDF prennent alors leur bâton de pèlerin pour arpenter les communes, provoquer des réunions publiques, convaincre les élus les plus réticents à revitaliser les anciens syndicats d'électrification ou à mettre en place de nouvelles structures. Ils peuvent compter sur un maillage territorial d'EDF encore très dense, ainsi que sur un réseau d'agents élus très actif, qui constitue dans certains cas un soutien précieux.

Grâce à cette « force de frappe » locale, le processus de refondation, qui pouvait apparaître assez risqué au moment où il allait être mis en œuvre, connaît un succès certain, que l'on peut mesurer à l'aune de deux principaux critères : le nombre de contrats signés, l'absence de réelle remise en question par les élus de la stratégie de refondation élaborée par EDF et la FNCCR. Le nombre de contrats de concession signés ne cesse d'augmenter durant la décennie 1990. Fin 1992, soit un an après la présentation du nouveau cahier des charges, on compte déjà une dizaine de signatures de contrats avec des syndicats regroupant en moyenne 97 \% des communes de leur département. Fin 1994, 56 \% des communes françaises ont signé le nouveau cahier des charges. Le chiffre s'élève à $84 \%$ fin 1997, soit $82 \%$ de la population. En moins d'une décennie, le nombre de syndicats départementaux d'électricité passe de moins d'une cinquantaine à près de 80 (Poupeau, 2004). Cependant, le processus est loin d'être achevé tant il se heurte à de multiples contingences locales, notamment des rivalités politiques. Plusieurs villes décident de conserver leur statut d'auto- 
rité concédante et de ne pas le transférer à un syndicat départemental. Au milieu des années 1990, près d'un millier de communes, représentant près du quart des usagers français, ont préféré contractualiser seules avec EDF (Bouvier, 2005). Dans la moitié des départements de France métropolitaine, les villes-centres se sont désolidarisées des syndicats, seuls $30 \%$ d'entre eux regroupant la totalité des communes de leur département. Bien que relancé par le nouveau cahier des charges, le processus de départementalisation reste donc inachevé.

Forte de ce constat mitigé, la FNCCR poursuit alors, par d'autres voies, son œuvre d'ingénierie et d'unification territoriale. La mise en œuvre de la directive européenne n² 2003/54 CE du 26 juin 2003 lui en donne l'opportunité. Grâce à ses appuis parlementaires, notamment au Sénat, la Fédération obtient, par l'article 33 de la loi du 7 décembre 2006 transposant le texte communautaire dans le droit français, un coup de pouce supplémentaire à son entreprise de départementalisation. L'article 33, qui est consacré aux aspects concessifs, stipule que « lorsque les attributions prévues par le présent article ne sont, pour les réseaux publics de distribution d'électricité, exercées ni par le département ni, au terme d'un délai d'un an suivant la date de publication de la loi n 2006-1537 du 7 décembre 2006 relative au secteur de l'énergie, par un unique syndicat de communes ou syndicat mixte sur l'ensemble du territoire départemental ou sur un ensemble de territoires départementaux contigus, le ou les représentants de l'État dans le ou les départements engagent, dans le cadre des dispositions prévues au $2^{\circ}$ du I de l'article L. 5211-5, la procédure de création d'un syndicat de communes ou d'un syndicat mixte pour l'exercice de ces compétences sur l'ensemble du territoire départemental ou sur un ensemble de territoires départementaux contigus ». En termes plus explicites, l'article de loi pousse à la départementalisation.

Cette disposition est appuyée peu de temps après par le ministère de l'Intérieur, lequel, par une circulaire, invite les préfets à constituer des syndicats départementaux. Deux actions conjointes sont alors menées. La première consiste à pousser les communes non adhérentes à rejoindre les structures départementales, grâce au bonus financier du contrat mais aussi d'autres incitations favorisées par le Fond d'amortissement des charges d'électrification (Facé). La seconde action vise à supprimer ou, à tout le moins, affaiblir les syndicats intercommunaux dits «primaires », qui pouvaient représenter les communes au sein des syndicats départementaux. Ces structures, constituées peu ou prou par cantons, conservaient parfois de fortes compétences (statut d'autorité concédante, maîtrise d'ouvrage en lieu et place des communes), ce qui pouvait entraver l'action des syndicats départementaux. Avec l'appui des préfets, les élus adhérents de la FNCCR s'efforcent de transférer ces prérogatives à l'échelon départemental, marginalisant de ce fait les structures intercommunales «primaires ». Dans certains syndicats, ces dernières se transforment en « comités territoriaux », qui deviennent désormais le lieu d'expression des besoins des communes, et non plus des organes décisionnaires.

Après quelques années de mise en œuvre de l'article 33, la départementalisation gagne à nouveau du terrain, comme le montre la carte suivante ${ }^{27}$ :

${ }^{27}$ D'après un document élaboré par la FNCCR. 
Figure 2 : Les syndicats d'énergie totalement ou partiellement départementalisés (2010)

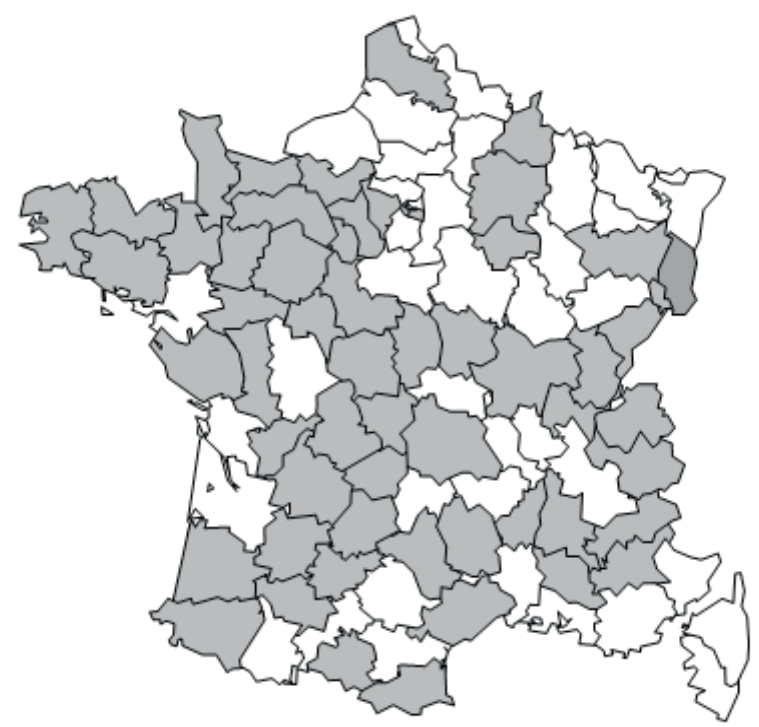

Fin 2010, 43 structures départementales maillent désormais le territoire national ${ }^{28}$. Toutes n'ont pas la même force de frappe. On peut distinguer trois groupes de syndicats aux profils très différents. Trois structures syndicales se détachent très nettement par l'ampleur de leurs moyens et de leurs compétences. Elles comptent plus de $100 \mathrm{M} €$ de budget et des effectifs qui varient entre 40 et 90 personnes. Le cas le plus emblématique d'un territoire profondément transformé par la contractualisation est celui de la Vendée. Alors que le SyDEV (syndicat départemental d'énergie et d'équipement de la Vendée) ne comptait début 1990 qu'un directeur détaché de la Direction départementale de l'agriculture et de la forêt (DDAF) et deux agents administratifs (Poupeau, 1999), il peut se targuer fin 2009 d'un budget de $140 \mathrm{M} €$, pour un effectif de 85 personnes. Son domaine de compétence n'a cessé de s'élargir. Il touche bien évidemment à l'électricité, à travers le contrôle de la concession mais aussi l'allocation des crédits d'électrification rurale, des actions en matière d'éclairage public (maintenance et exploitation), d'achat d'énergie pour le compte des communes ou de maîtrise de la demande d'énergie (diagnostics de bâtiments, information et sensibilisation des citoyens). En 2002, le SyDEV crée la REVe (Régie d'Électricité de Vendée) afin de développer les énergies renouvelables. En 2014, Vendée Énergie (qui s’est substituée en 2012 à la REVe) est une société d'économie mixte qui exploite des parcs éoliens et soutient le développement du photovoltaïque sur toiture. Par ailleurs, le syndicat a élargi ses compétences dans le gaz, en acquérant le statut d'autorité concédante pour le compte des 100 communes vendéennes qui bénéficient d'une desserte. Il a enfin joué un rôle moteur dans la mise en place de «Smart grid Vendée », un projet d'expérimentation de réseaux intelligents. Cette stratégie de diversification n'est pas l'apanage du seul SyDEV, comme en

${ }^{28}$ SEA Conseil en stratégie, Altime Charles Riley, «La distribution d'électricité en France. Quelles évolutions? Organisation territoriale de l'énergie et évolution des métiers », janvier 2011. Les données globales qui suivent sur les syndicats sont extraites de cette étude. 
témoigne l'ex SCBPE qui s'est transformé en 1997 en Sippérec (Syndicat intercommunal de la périphérie de Paris pour l'électricité et les communications), à la suite d'une décision des élus de lui confier une nouvelle tâche : le développement des réseaux de fibre optique pour l'aménagement numérique de leur territoire. Tout comme le SyDEV, le Sippérec se présente aujourd'hui comme l'un des syndicats les plus dynamiques, non pas simplement au plan local mais aussi au plan national, dans la mesure où certaines de ses actions ont fait évoluer fortement le cadre réglementaire français (Bellanger, Poupeau, 2013).

Il ne faudrait pas toutefois assimiler la situation de l'ensemble des syndicats départementaux à ces deux cas emblématiques. De nombreuses intercommunalités en restent encore à un stade émergent de structuration et de développement. La plupart des syndicats sont bien moins organisés et puissants que ne le sont le SyDEV ou le Sippérec. Deux autres groupes peuvent être identifiés. Le premier est formé de 25 structures environ, qui ont un budget compris entre 30 et $95 \mathrm{M} €$, avec un effectif variant entre 10 et 60 personnes. Il correspond aux territoires dans lesquels la départementalisation commence à produire aujourd'hui seulement ses effets, avec des premières formes de diversification au-delà du contrôle de la concession d'électricité. Pour 31 autres syndicats, dont les ressources sont en-deçà des $30 \mathrm{M} €$ et de 30 personnes, il serait abusif de parler d'acteurs locaux forts. Ces structures, de création récente ou qui en sont à parachever leur départementalisation, restent en quête d'une vraie stratégie leur permettant de devenir des acteurs aussi incontournables que ne le sont le SyDEV ou le Sippérec.

\section{Conclusion}

En l'espace d'une vingtaine d'années, le paysage institutionnel local de la distributionfourniture (jusqu'en 2007) puis de la distribution publique d'énergie ${ }^{29}$ a profondément évolué. Parmi les éléments qui ont contribué à ces mutations, les contrats de concession ont joué un rôle majeur. Véritables instruments d'action publique utilisés tant par EDF que par les élus locaux, ils ont eu pour effet de restructurer l'espace de la négociation locale et les rapports de force entre acteurs, notamment entre mondes rural et urbain. Ils ont permis de maintenir une forme de solidarité territoriale que le processus de libéralisation menaçait - et menace encore aujourd'hui - de mettre à mal. La mobilisation des contrats a ainsi participé de ce que le géographe Robert David Sack appelle un processus de territorialisation (Sack, 1986), par lequel un ou plusieurs acteurs mettent en place des dispositifs qui visent à contrôler une population ou gérer un problème politique à l'intérieur d'une zone géographique qu'ils délimitent, légitiment et régulent ${ }^{30}$. Pour la FNCCR, il s'agit de préserver un modèle de service public qui, bien que critiqué pour sa centralisation, procure à cette association une place privilégiée et lui permet de maintenir l'influence du monde rural, contre les villes.

\footnotetext{
${ }^{29}$ Rappelons qu'en 2007, le marché de l'électricité a été totalement ouvert à la concurrence, ce qui a eu pour effet de sortir l'activité de fourniture du champ de la concession communale (excepté pour les tarifs réglementés de vente, au caractère a priori éphémère). Celle-ci se limite donc désormais à la partie « distribution », i.e. acheminement.

${ }^{30}$ " Territoriality will be defined as the attempt by an individual or a group to affect, influence, or control people, phenomena, and relationships, by delimitating and asserting control over a geographic area. This area will be called territory » (Sack, 1986:19).
} 
Pour EDF, le contrat répond à un autre souci, constant dans les années 1990 : retarder le plus longtemps possible les conséquences d'une libéralisation des marchés de l'énergie et façonner le futur espace concurrentiel. Fruit d'une convergence d'intérêts entre ces deux acteurs, le dispositif contractuel, composé d'un cahier des charges et de documents complémentaires (comme le «protocole grandes villes »), est ainsi mobilisé au service d'une véritable stratégie d' "ingénierie institutionnelle », qui vise à contenir les menaces qu'un certain nombre d'acteurs à la fois transnationaux, nationaux et locaux font peser contre la pérennité des arrangements politiques d'après-guerre bâtis autour du modèle de service public.

Cette forme d'ingénierie repose sur une série de mécanismes que nous nous sommes attachés à mettre en évidence dans cette contribution : définition, au sein de l'entreprise contractante, de l'univers des possibles imposé aux futurs négociateurs par fixation / exclusion de certains sujets et volonté de cadrage financier (rejet d'une « dépéréquation » tarifaire, définition du niveau de la rente redistribuée vers les collectivités locales); sélection en amont, toujours par l'entreprise contractante, d'un partenaire de négociation exclusif (la FNCCR) partageant son souci de stabilisation du secteur de la distribution-fourniture (puis de la seule distribution); mise au point de mesures incitatives (notamment financières) permettant d' « enrôler » les acteurs locaux autour d'un cahier des charges pré-défini ; création d'une forme d'interdépendance (clause de la concession la plus favorisée) entre les contrats signés localement pour obliger les acteurs locaux à en référer constamment à leurs services centraux ; stratégie de mise en œuvre permettant de sélectionner les territoires les plus favorables pour lancer une dynamique nationale... Analyser ainsi le traité de concession, dans la longue durée, comme porteur d'un « script» (Akrich, 1987) qui performe l'action, structure l'espace économique et institutionnel présente un intérêt heuristique certain, même s'il s'agit, en l'espèce, d'un type de contrat assez particulier, qui ne ressemble que partiellement aux formes classiques de la gestion déléguée ${ }^{31}$ (eau, assainissement, transports publics...). Une telle approche permet de révéler les intentions cachées des acteurs derrière des articles, des clauses, des annexes financières a priori «techniques » mais qui contiennent, sans le dire, un projet institutionnel implicite. Ce faisant, et c'est là l'un des enseignements majeurs de cet exemple qui peut inspirer d'autres travaux, il apparaît que le contrat ne saurait être simplement considéré comme organisant des rapports de coopération entre acteurs. Il peut être également mobilisé en vue de (re)structurer un secteur, en discriminant les acteurs qui participent à sa régulation, en redistribuant des ressources, en clôturant l'espace politique. Il peut ainsi devenir un opérateur de rehiérarchisation institutionnelle dont les effets peuvent se faire ressentir sur un plus long terme, comme c'est le cas en matière de gouvernance locale des réseaux d'énergie ${ }^{32}$.

\footnotetext{
${ }^{31}$ Le rapport de la Cour des comptes de 2013 rappelle le caractère quelque peu atypique de la distribution publique d'électricité en France, qui repose sur un régime de la concession dans lequel le concessionnaire est imposé (ERDF, filiale d'EDF) et où les tarifs sont fixés au plan national (Cour des comptes, "Les concessions de distribution d'électricité : une organisation à simplifier, des investissements à financer », Rapport annuel 2013, pages 105-217).

32 II aurait été trop long d'aborder dans cet article la période ultra-contemporaine et les lois MAPTAM (Modernisation de l'action publique territoriale et d'affirmation des métropoles), NOTRe (Nouvelle organisation territoriale de la République) et Transition énergétique pour la croissance verte, votées respectivement les 27 janvier 2014, 7 août et 17 août 2015. Bien que renforçant les compétences des nouvelles métropoles en matière de distribution publique d'énergie, ces trois lois ne menacent que partiellement les grands syndicats départementaux. Sur ce point, cf Poupeau, $2015 \mathrm{~b}$.
} 


\section{Bibliographie}

AKERLOF G. A., (1970). The Market for "Lemons": Quality Uncertainty and the Market Mechanism. Quarterly Journal of Economics 84 (3), 488-500.

AKRICH M., (1987). Comment décrire les objets techniques? Techniques et culture 9, 49-64.

AUBY J.-F., (1995). La délégation de service public. PUF, Paris.

BARNETT R. E., (1992). The Sound of Silence: Default Rules and Contractual Consent.Virginia Law Review 78 (4), 821-911.

BELLANGER E. et POUPEAU F.-M., (2013). Lumières sur la banlieue. Histoire du syndicat intercommunal de la périphérie de Paris pour l'électricité et les réseaux de communication, (SIPPEREC). Les Editions de l'Atelier, Paris.

BOITEUX M., (1993). Haute Tension. Éditions Odile Jacob, Paris.

BOURJOL M. et LE LAMER C., (1984). Énergie et décentralisation. Economica, Paris.

BOUVIER G., (2005). Les collectivités locales et l'électricité. Territoires, acteurs et enjeux autour du service public local de l'électricité en France. Thèse de géographie, Université Paris 8.

BROUSSEAU E., (2000). Contrat ou Confiance, Confiance et Contrat In AUBERT F., SYLVESTRE J.-P., (dir). Confiance et Rationalité, INRA Edition, Les Colloques 97, 65-80.

CNFPT (ed.), (1993). Distribution d'énergie et décentralisation. Quels nouveaux enjeux pour les collectivités locales? Les éditions du CNFPT, Paris.

GABILLET P., (2015). Les entreprises locales de distribution à Grenoble et Metz. Des outils de gouvernement énergétique urbain partiellement appropriés. Thèse de doctorat d'aménagement de l'espace et d'urbanisme, Université Paris-Est.

HALPERN C., LASCOUMES P. et LE GALES P., (dir.), (2014). L'instrumentation de l'action publique. Controverses, résistances, effets. Les Presses de Sciences Po, Paris.

HART O. and MOORE J., (1988). Incomplete Contracts and Renegotiation. Econometrica, 56 (4), 755-785.

LAFFONT J.-J. and TIROLE, J., (1993). A Theory of Incentives in Procurement and Regulation. MIT Press, Cambridge.

LASCOUMES P. et LE GALES P., (dir.), (2004). Gouverner par les instruments. Les Presses de Sciences Po, Paris.

LASCOUMES P. et SIMARD L., (2011). L'action publique au prisme de ses instruments. Introduction. Revue française de science politique 61 (1), 5-22.

LENOIR D., (2007). Énergie, changeons de cap! Scénario pour une France durable. Terre Vivante, Mens.

LEVI-FAUR D., (1999). The Governance of Competition: the Interplay of Technology, Economics, and Politics in European Union Electricity and Telecom Regimes. Journal of Public Policy 19 (2), 175-207.

LORRAIN D., (1991). De l'administration républicaine au gouvernement urbain. Sociologie du Travail 4, 461-484.

LORRAIN D., (1993). Après la décentralisation, l'action publique flexible. Sociologie du Travail 35 (3), $285-307$.

NEUVILLE J.-P., (1996). Le contrat de confiance, Etude des mécanismes de coopération dans le partenariat industriel autour de deux grands constructeurs automobiles européens, Thèse de doctorat, Institut d'Études Politiques de Paris.

PALARD J., (1996). Les associations d'élus locaux: amicales ou groupes de pression? In Institut de la Décentralisation, La décentralisation en France. L'état des politiques publiques, la dynamique des réformes locales, la dimension européenne, La Découverte, Paris, 115-123.

PETAUX J., (1994). L'école des maires. Les associations d'élus locaux. Politix 28, 49-63.

POUPEAU F.-M., (1999). EDF ou la permanence d'un compromis républicain. Le système de distribution électrique français entre État et collectivités locales, de la nationalisation à la mondialisation. Thèse de doctorat, Institut d'Études Politiques de Paris.

POUPEAU F.-M., (2004). Le service public à la française face aux pouvoirs locaux. Les métamorphoses de l'État jacobin. CNRS Éditions, Paris. 
POUPEAU F.-M., (2007). La fabrique d'une solidarité territoriale. État et élus ruraux dans l'adoption d'une péréquation des tarifs de l'électricité en France. Revue française de science politique 57 (5), 599-628.

POUPEAU F.-M., (2015a). L'électricité et les pouvoirs locaux en France. Une autre histoire du service public (1880-1980). Tome II du mémoire de HDR, Université Paris-Est.

POUPEAU F.-M., (2015b). La gouvernance locale des réseaux d'énergie. Entre départementalisation et métropolisation In MARCOU G., EILLER, A.-C., POUPEAU F.-M., STAROPOLI C., Gouvernance et innovations dans le système énergétique : quel rôle pour les collectivités territoriales? L'Harmattan, Paris, 103-119.

ROUBIEU O., (1994). Le modèle du manager. L'imposition d'une figure légitime parmi les hauts fonctionnaires des collectivités locales. Politix 28, 35-48.

SACK R. D., (1986). Human Territoriality. Its Theory and History. Cambridge University Press, Cambridge.

SCHMIDT S. K., (1997). Sterile Debates and Dubious Generalisations : European Integration Theory Tested by Telecommunications and Electricity. Journal of Public Policy 16 (3), 233-271.

SCHMIDT S. K., (1998). Commission Activism: Subsuming Telecommunications and Electricity under European Competition Law. Journal of European Public Policy 5 (1), 169-184.

SPENCE M., (1973). Job Market Signaling. Quarterly Journal of Economics 87 (3), 355-374.

STIGLITZ J. E. and WEISS A., (1981). Credit Rationing in Markets with Imperfect Information. American Economic Review 71, 393-410

STOFFAES C., (dir.), (1994). Entre monopole et concurrence, La régulation de l'énergie en perspective historique. Editions PAU, Paris.

WIEVIORKA M. et TRINH S., (1989). Le modèle EDF. La Découverte, Paris. 


\section{Annexe 1 Méthodologie et utilisation des données}

Cet article s'appuie sur une observation au long cours du système énergétique français, entamée à partir d'une thèse soutenue en 1999 (Poupeau, 1999). Ce premier travail s'est appuyé sur une série d'entretiens réalisés avec des acteurs d'EDF ayant participé au processus de contractualisation au début des années 1990. Ces interviews ayant été menées de manière anonyme, nous avons opté pour ne pas donner ici les noms des personnes impliquées dans ce processus, hormis les dirigeants de l'entreprise (PDG, Directeur général) ou d'associations d'élus qui ont pris des décisions publiques en la matière. La mention des documents internes à EDF permettra aux historiens qui voudraient retravailler sur le sujet de retrouver la trace des individus impliqués dans ce processus et les débats qu'ils ont pu avoir à cette occasion. Ici nous intéresse, en tant que politiste, la question du processus de contractualisation et de ses conséquences sur le paysage institutionnel local, angle d'attaque qui nécessite de regarder de plus près les pratiques des acteurs, en contrepartie de la garantie d'une certaine anonymisation de leur témoignage. À noter que la thèse soutenue en 1999 prévoyant un « embargo » des données pendant dix ans (sauf autorisation expresse de l'entreprise, qui a été obtenue s'agissant de productions scientifiques antérieures), les informations ici récoltées appartiennent désormais au « domaine public ».

Cette recherche inaugurale a été ensuite complétée par plusieurs enquêtes ou observations diverses :

- un post-doctorat mené en 2000-2001 à l'Institut d'Études Politiques de Paris sur une comparaison de l'action des collectivités locales françaises en matière de politique énergétique et de télécoms hauts débits (qui a donné lieu à un ouvrage, Poupeau, 2004)

- le suivi de nombreuses enquêtes (notamment pour le Plan Urbanisme Construction Architecture du Ministère de l'écologie et du développement durable) sur les politiques énergétiques locales menées par les villes, départements ou intercommunalités (2004-2010)

- une recherche, menée conjointement avec un collègue historien de Paris-1, sur l'histoire, des origines à nos jours, du SCBPE (Syndicat des communes de la banlieue parisienne pour l'électricité), qui s'est transformé en 1997 en SIPPEREC (Syndicat intercommunal de la périphérie de Paris pour l'électricité et les communications) (2011-2013) (Bellanger, Poupeau, 2013)

- de nombreux échanges avec les acteurs du système énergétique français, notamment des représentants d'associations d'élus

- l'animation d'une formation continue à l'Université Paris-Ouest Nanterre La Défense destinée à des agents de maitrise d'EDF souhaitant passer cadre, et qui a été l'occasion de recueillir des informations sur l'évolution de la «géopolitique locale de l'énergie » (2002-2015)

- enfin, la participation (2013), en tant qu'expert, au comité national des experts ayant été associé à la préparation du projet de loi sur la transition énergétique et la croissance verte, qui a été votée le 17 août 2015 par le Parlement. 


\section{Annexe 2}

\section{Formules de calcul des redevances du modèle de cahier des charges de concession pour la distribution publique d'électricité ${ }^{33}$ (1992)}

\section{$\mathrm{R} 1$, redevance dite de fonctionnement.}

$\mathrm{R} 1=\left[75 \mathrm{~L}_{\mathrm{C}}+0,7 \mathrm{P}_{\mathrm{C}}\right] \times \mathrm{C} \times\left[1+\mathrm{P}_{\mathrm{C}} / \mathrm{P}_{\mathrm{D}}\right] \times[0,01 \mathrm{D}+0,75] \times\left[0,15+0,85 \times \mathrm{ING} / \mathrm{ING}_{0}\right]$

LC : longueur, en km, au 31 décembre de l'année précédente, des réseaux HT et BT concedes de la concession.

PDU : population municipale de l'ensemble des communes urbaines desservies par EDF dans le département où se situe la concession.

PC : population municipale de la concession.

PD : population municipale desservie par EDF dans le département où se situe la concession. D : durée de la concession (exprimée en années et comprise entre 20 et 30 ans).

ING : valeur de l'index « ingénierie » (édité par le ministère de l'équipement et du logement) du mois de décembre de l'année précédente.

ING0 : valeur de l'index « ingénierie » du mois de décembre de l'année précédant celle de la signature du contrat de concession.

Sachant que le terme $\mathrm{C}$ dépend lui aussi de la population de la concession.

$\mathrm{C}=1$ si la population est au moins égale à 150000 habitants.

$\mathrm{C}=0,2+\mathrm{PC} / \mathrm{PDU}$ x 0,8 si la population de la concession PC est inférieure à 150000 habitants et si la population urbaine départementale PDU est inférieure à 150000 habitants. $\mathrm{C}=0,2+\mathrm{PC} / 150000 \times 0,8$ si la population de la concession PC est inférieure à 150000 habitants et si la population urbaine départementale PDU est au moins égale à 150000 habitants.

Il est prévu que R1 ne soit pas inférieur au montant maximum de la redevance des frais de contrôle et qu'il soit limité par la formule : 2500000 x [0,15 + 0,85 x ING/ ING0] francs.

\section{$R 2$, redevance dite d'investissement}

$\mathrm{R} 2=(0,74 \mathrm{~B}+0,30 \mathrm{E}-0,5 \mathrm{~T})(1+\mathrm{PC} / \mathrm{PD}) \times(0,005 \mathrm{D}+0,125)$.

B : montant total hors TVA mandaté au cours de l'année pénultième par la collectivité maître d'ouvrage, des travaux réalisés par celle-ci sur le réseau concédé.

Ce montant est déterminé à partir des attestations établies par la collectivité maître d'ouvrage en vue du reversement par le concessionnaire à celle-ci, dans les conditions prévues par le décret du 7 octobre 1968, de la TVA ayant grevé le coût des travaux, et après défalcation des montants versés par le concessionnaire au titre de l'abondement des dépenses effectuées par la collectivité en vue d'améliorer l'esthétique des ouvrages, suivant les modalités prévues à l'article 3 ci-après.

33 « Modèle de cahier des charges de concession pour le service public de la distribution d'énergie électrique », juin 1992, annexe I, article 2 « Redevance de concession ». 
E : montant total hors TVA en francs des travaux d'investissement sur les installations d'éclairage public, mandaté par la collectivité maître d'ouvrage l'année pénultième.

Ce montant est déterminé par un état dressé par l'autorité concédante explicitant la situation, la nature et le montant des travaux réalisés.

T, produit net de la taxe municipale sur l'électricité sur le territoire de la concession, ayant fait l'objet de titres de recettes de l'autorité concédante l'année pénultième.

D, durée de la concession (exprimée en années et comprise entre 20 et 30 ans).

PD : population municipale desservie par EDF dans le département où se situe la concession.

PC : population municipale de la concession. 